\title{
YAP is closely correlated with castration-resistant prostate cancer, and downregulation of YAP reduces proliferation and induces apoptosis of $\mathrm{PC}-3$ cells
}

\author{
XIA SHENG $^{1 *}$, WEN-BIN LI ${ }^{1 *}$, DE-LIN WANG ${ }^{1}$, KE-HONG CHEN ${ }^{1}$, JIAN-JIA CAO ${ }^{1}$, \\ ZHAO LUO ${ }^{1}$, JIANG HE ${ }^{2}$, MEI-CAI LI ${ }^{1}$, WU-JIANG LIU ${ }^{3}$ and CHAO YU ${ }^{4}$ \\ ${ }^{1}$ Department of Urology, The First Affiliated Hospital of Chongqing Medical University, Chongqing 400016; \\ ${ }^{2}$ Department of Urology, University-Town Hospital of Chongqing Medical University, Chongqing 401331; \\ ${ }^{3}$ Department of Urology, Institute of Urology, First Hospital of Peking University, Beijing 100034; \\ ${ }^{4}$ Department of Surgery, Life Science Research Institute of Chongqing Medical University, \\ Chongqing 400016, P.R. China
}

Received August 19, 2014; Accepted May 19, 2015

DOI: $10.3892 / \mathrm{mmr} .2015 .4005$

\begin{abstract}
Yes-associated protein 65 (YAP65) has been implicated as an oncogene, and its expression is increased in human cancer. Previous studies have demonstrated that alterations in YAP activity may result in tumourigenesis of the prostate. With androgen deprivation therapies becoming progressively ineffective, often leading to life-threatening androgen-resistant prostate cancer (CRPC). The present study aimed to analyse the role of YAP in prostate cancer (PCa), particularly in CRPC. YAP protein was detected using immunohistochemistry and western blot analysis in different prostatic tissues. In addition, three specific RNA interference vectors targeting the human YAP gene were synthesised, and PC-3 cells with a stable inhibition of YAP were obtained by transfection. MTT, flow cytometry, reverse transcription-quantitative polymerase chain reaction and western blot assays were used to analyse the effects of YAP inhibition on the proliferation and apoptosis of PC-3 cells. The frequency of cells that were positive for YAP protein in $\mathrm{PCa}(78.13 \%)$ was significantly higher, compared with para-PCa $(26.67 \%$; $\mathrm{P}=0.007)$ and benign prostatic hyperplasia $(0 \% ; \mathrm{P}=0.002)$. The frequency of cells, which were positive for the expression of YAP exhibited a positive correlation $(\mathrm{P}=0.008)$ with the Gleason score, the tumour-node-metastasis staging $(\mathrm{P}=0.033)$ and the level of prostate specific antigens $(\mathrm{P}=0.0032)$ in $\mathrm{PCa}$. The proliferative capacity of the trans-
\end{abstract}

Correspondence to: Professor De-Lin Wang, Department of Urology, The First Affiliated Hospital of Chongqing Medical University, 1 Medical College Road, Chongqing 400016, P.R. China E-mail: dlwangws@sina.com

\section{${ }^{*}$ Contributed equally}

Key words: Yes-associated protein, TEA domain family member 1, prostate cancer, PC-3 cell, RNA interference fected group was significantly lower, compared with the negative control group $(\mathrm{P}=0.022)$. The cell-cycle of the transfected group was arrested in the G1 stage, which was detected using flow cytometry, and there was a significant increase in the apoptosis of cells in the transfected group $(\mathrm{P}=0.002)$. The mRNA and protein levels of TEA domain family member 1 were inhibited in the transfected group $(\mathrm{P}=0.001$ and $\mathrm{P}=0.00$, respectively). Therefore, it was concluded that gene transcription and protein expression of YAP may be involved in the development of PCa, particularly CRPC, and may be a novel biomarker for investigation of the occurrence and progression of CRPC. However, the mechanism underlying the modulation of YAP in CRPC remains to be fully elucidated.

\section{Introduction}

The body and organ size of a mammal is determined by the number and size of cells; however, the total cell mass is not always adjusted toward the normal size if it is experimentally or accidentally perturbed (1). Previous genetic screens of Drosophila have revealed that the Hippo signalling pathway is critical in restricting organ size by controlling cell cycle exit and cell death $(2,3)$. The Hippo pathway restricts cell growth and proliferation and promotes apoptosis by regulating the nuclear localisation of Yes-associated protein (YAP) and TEA domain family member (TEAD) in mammals (4). This regulation is achieved by the transcriptional activation of the Hippo pathway target genes, including cyclin E, diap1, and bantam microRNA (5-7). YAP, a 65-kDa protein, is a transcriptional co-activator of several transcription factors via its own WW-domain. It is also a potent growth promoter, which has been identified as an oncogenic protein in mammalian cells (8-10). The TEAD family of transcription factors is considered to be a major partner of YAP and TAZ in the Hippo pathway (11). Substantial evidence has revealed that TAED1 and YAP share a substantial number of target genes (12-14). In support of this evidence, TEAD1 and TEAD2 double-knockout-mice have been observed to exhibit 
phenotypes similar to those of YAP-knockout mice (15). Furthermore, ablation of the expression of TAED decreases the ability of YAP/TAZ to promote anchorage independent growth $(12,16)$. Despite its conservation and close association with cancer, the Hippo pathway has not been systematically investigated in mammalian cells.

Prostate cancer $(\mathrm{PCa})$ is a malignant carcinoma with one of the highest morbidity rates worldwide, primarily endangering the health of aging males (17), particularly castration-resistant prostate cancer (CRPC). The treatment options for patients with CRPC remain limited. Although the mechanisms involved in the occurrence and development of CRPC remain to be elucidated, it has been observed that dysregulation of the Hippo signalling pathway is important in the proliferation of tumour cells, and the activation of YAP gives rise to carcinoma $(5,18)$. YAP is considered to be the key component downstream of the Hippo signalling pathway, and the importance of Hippo signalling in controlling mammalian organ size has been investigated extensively in the liver, where transgenic overexpression of YAP leads to hepatomegaly (19). The overexpression of YAP has also been observed in gastric cancer $(20)$ and in PCa $(21,22)$. However, the function of YAP in CRPC cells remains to be elucidated.

Studies have revealed that the Hippo signalling pathway is involved in cell cycle regulation (23). The dysregulation of this pathway, which leads to YAP activation, induces oncogenic transformation in cooperation with distinct transcription factors, including TEAD family members (24). In the present study, PCa specimens were obtained to perform analyses of the correlation between YAP, and the staging and grading of the clinical pathology, Gleason score and level of prostate specific antigen (PSA) in the PCa cells. In addition, the PC-3 CRPC cell line was selected to further investigate YAP in vitro. The pMagic7.1-RNA interference-YAP-1, 2, 3 plasmid was used to inhibit YAP, to determine the effect of YAP inhibition on the proliferation and apoptosis of the PC-3 cells. The differences in the expression of YAP and TEAD1 following transfection were also analysed to determine the role of the Hippo signalling pathway in PC-3 cells.

\section{Materials and methods}

Specimen collection. Tissue specimens used in the present study were acquired from the First Affiliated Hospital of Chongqing Medical University (Chongqing, China) between March 2009 and July 2012. The study was approved by the Ethics Committee of Chongqing Medical University, and informed, written consent regarding the use of tissues was obtained from all patients. There were 62 male patients in total, with an age range from 53-81 years old. A total of $32 \mathrm{PCa}$ samples were obtained during surgery. Certain specimens were collected from patients with dysuria following drug and surgical castration. Furthermore, 15 samples of benign prostatic hyperplasia (BPH) tissue from transurethral resection of the prostate were obtained as a control, and 15 samples of para-prostate carcinoma tissue (para-PCa) were also obtained during surgery. All specimens were confirmed pathologically and stored at $-80^{\circ} \mathrm{C}$. However, PCa and CRPC cannot be distinguished by pathological diagnosis. The ages of the patients with PCa ranged between 53 and 81 years (mean,
67). According to Gleason's grading system (25), there were eight cases with scores of $\leq 6,11$ cases with scores of 7 and 13 cases with scores $>8$. The tumour-node-metastasis (TNM) staging (26) indicated that there were 15 cases of T1-T2 and 17 cases of T3-T4. In addition, nine cases had metastases.

Immunohistochemistry (IHC). The specimens were fixed with $10 \%$ formaldehyde (ZSGB-Bio, Beijing, China), cut into sections $(5 \mu \mathrm{m})$ and stained according to the streptomycin anti-biotin-peroxidase two-step method (27). Briefly, following gradient alcohol dehydration, the sections were incubated in $10 \%$ hydrogen peroxide for $10 \mathrm{~min}$ and antigen retrieval was performed using a microwave vacuum histo-processor (RHS-1; Milestone, Sorisole, Italy) at $121^{\circ} \mathrm{C}$ for $15 \mathrm{~min}$. Following blocking with goat serum (Gibco-BRL, Carlsbad, CA, USA), the goat anti-rabbit monoclonal primary antibody (1:200; Santa Cruz Biotechnology, Inc., Dallas, TX, USA) and goat anti-rabbit IgG horseradish peroxidase (HRP)-labeled secondary antibody (ZSGB-Bio) were added for $1 \mathrm{~h}$ at $37^{\circ} \mathrm{C}$. Following 3,3'-diaminobenzidine and hematoxylin (ZSGB-Bio) treatment, the sections were sealed with neutral resin (ZSGB-Bio) and observed under an inverted microscope (LW300-38LF; Bio-Rad Laboratories, Inc., Hercules, CA, USA).

A semiquantitative scoring criterion for YAP in the tissue specimens was used. Tissue sections were observed under an inverted microscope, and staining intensity and positive areas were recorded. The staining intensity was evaluated on a scale between 0 and 3 ( 0 , negative; 1 , weak; 2 , moderate; 3 , strong). The percentage of positive areas was scored using a four-tier system $(0,0 \% ; 1, \leq 25 \% ; 2, \leq 50 \% ; 3, \leq 100 \%)$. The intensity of the immunoreaction was calculated from the score of the staining intensity and the percentage of positive areas. An intensity of 0-1 was negative, 2-4 was weak positive, 5-6 was medium positive and 7-8 was strong positive.

Cell culture. The PC-3 cells, obtained from osseous metastasis of the prostate cancer of an elderly man, were purchased from the Shanghai Cell Bank of the Chinese Academy of Sciences (Shanghai, China), and was a CRPC cell line (28). The cells were cultured in a mixture of Dulbecco's modified Eagle's medium (DMEM) and F12 (1:1; HyClone Laboratories, Inc., South Logan, UT, USA), containing $10 \%$ fetal bovine serum (FBS; Gibco-BRL), in an incubator at $37^{\circ} \mathrm{C}$ with $5 \% \mathrm{CO}_{2}$.

Structure of YAP-RNAi. Using the sequence of the YAP gene (NM_006106) from GenBank (http://www.ncbi.nlm.nih.gov/ gene/?term=YAP+NM-006106), the Shanghai SBO Medical Biotechnology Co., Ltd. website and online RNA screening technology (http://www.sbo-bio.com.cn), the interference sequences were designed as follows: RNAi-1, forward 5'-CCG GGCTCATTCCTCTCCAGCTTCTCAAGAGAAAGCTGG AGAGGAATGAGCTTTTTTG-3' and reverse 5'-AATTCA AAAAAGCTCATTCCTCTCCAGCTTTCTCTTGAGAAG CTGGAGAGGAATGAGC-3'; RNAi-2, forward 5'-CCGGCT TAACAGTGGCACCTATTTCAAGAGAATAGGTGCCAC TGTTAAGGTTTTTTG-3' and reverse 5'-AATTCAAAA AACCTTAACAGTGGCACCTATTCTCTTGAAATAGGT GCCACTGTTAAGG-3'; RNAi-3, forward 5'-CCGGCCGTT TCCCAGACTACCTTCTCAAGAGAAAGGTAGTCTGGG 
AAACGGTTTTTTG-3' and reverse 5'-AATTCAAAAAAC CGTTTCCCAGACTACCTTTCTCTTGAGAAGGTAGTC TGGGAAACGG-3'; negative control, forward 5'-FCCGGT TCTCCGAACGTGTCACGTTTCAAGAGAACGTGACAC GTTCGGAGAATTTTTG-3' and 5'-reverse AATTCAAAA ATT CTCCGAACGTGTCACGTTCTCTTGAAACGTGAC ACGTTCGGAGAA-3'.

The forward sequences were compared using BLAST (http://www.ncbi.nlm.nih.gov/BLAST) to the published Expressed Sequence Tags database, and the three pairs of specific sequences were confirmed in addition to the human YAP genes. No genetic homology with other genes was found. The three pairs of RNAi oligonucleotides targeting human YAP mRNA and the control sequence were synthesised at SBO-Bio (Shanghai, China) (29).

A total of three pairs of RNAi plasmid vectors, pMagic7.1-Puro/green fluorescent protein (GFP)-RNAi-YAP 1,2 , and 3 , and a negative control (NC) vector were constructed (Beijing ComWin Biotech Co.,Ltd, Beijing, China). Three pairs of plasmids and the NC vector were transfected into the PC-3 prostate cancer cells using Lipofectamine 2000 (Invitrogen Life Technologies, Carlsbad, CA, USA). Non-transfected (NT) PC-3 cells were used as a control.

Stable YAP-inhibition in PC-3 cells. On the day prior to transfection, $\mathrm{PC}-3$ cells in the logarithmic growth stage were transferred into 6-well plates $\left(2 \times 10^{5}\right.$ cells/well), and $2 \mathrm{ml}$ DMEM/F12 containing $10 \%$ FBS was added. The plates were incubated overnight at $37^{\circ} \mathrm{C}$ to ensure that the cells occupied $\sim 90 \%$ of the well. Transfection was performed using Lipofectamine 2000, according to the manufacturer's instructions. Green fluorescence was observed using an inverted fluorescence microscope (LW300-38LF; Bio-Rad Laboratories, Inc.) 48 h after transfection. Puromycin (Invitrogen Life Technologies) was added $(1 \mathrm{mg} / \mathrm{l}$ per well) to the plates for screening of stable cell lines for 2 days at $37^{\circ} \mathrm{C}$, during which several cells underwent apoptosis, and the medium was replaced without puromycin. The cell clusters exhibiting green fluorescence were selected and inoculated into flasks. The NT group acted as a control. The medium was replaced every other day. After 2 weeks, the clusters of cells were observed under an inverted fluorescence microscope and, after 1 month, cells were obtained, which were observed to be stably expressing YAP inhibition (29).

Reverse transcription-quantitative polymerase chain reaction $(R T-q P C R)$. Total RNA was extracted from each group of cells using RNAiso reagent (Tiangen Biotech Co., Ltd., Beijing, China). The total RNA was quantified using a UV spectrophotometer (UV2800; Bio-Rad Laboratories, Inc.), and $1 \mu \mathrm{g}$ RNA was used for the RT reaction to obtain cDNA. The qPCR reactions were performed with $0.5 \mu \mathrm{g}$ DNA to amplify the YAP gene, with GAPDH as the internal reference using the ABI 2720/2700 Cycler (Applied Biosystems Life Technologies, Foster City, CA, USA). The cycling conditions were as follows: $94^{\circ} \mathrm{C}$ for $30 \mathrm{sec}, 55^{\circ} \mathrm{C}$ for $30 \mathrm{sec}$ and $72^{\circ} \mathrm{C}$ and $45 \mathrm{sec}$ (30 cycles). The primer sequences for the YAP mRNA obtained from Beyotime Institute of Biotechnology were as follows: Forward 5-TGAACA AACGTCCAGCAAGATAC-3 and reverse 5-CAGCCCCCA AAATGAACAGTAG-3. The target fragment was $165 \mathrm{bp}$. Primer sequences for the TEAD1 mRNA were as follows: Forward
5-TGAATCAGTGGACATTCGTCA-3 and reverse 5-GCCATT CTCAAACCTTGCATA-3. The target fragment was $280 \mathrm{bp}$. Primer sequences for GAPDH mRNA were as follows: Forward 5-ACCACCATGGAGAAGGCTGG-3 and reverse 5-CTCAGT GTAGCCCAGGATGC-3. The target fragment was $500 \mathrm{bp}$. The PCR products were analysed using agarose gel electrophoresis (Sino-American Biotechnology Co., Ltd., Luoyang, China) with $100 \mathrm{~V}$ and then $300 \mathrm{~mA}$ and then were visualised under UV light. A Bio-Rad gel formatter (Bio-Rad Laboratories, Inc.) was used to analyse the original band (30).

Western blot analysis. The cells of different prostatic tissues, three stable transfection experimental groups, the NC group, and the NT group were collected. Cell lysates were prepared using a mixture of phenylmethylsulfonyl fluoride and protease inhibitor (1:99; Beyotime Institute of Biotechnology, Shanghai, China). The proteins were separated by gel electrophoresis and then transferred onto $0.45 \mu \mathrm{m}$ polyvinylidene fluoride membranes (Beyotime Institute of Biotechnology). The mouse anti-rabbit primary antibody (1:200) and HRP-labeled secondary antibody $(1: 5,000)$ were added for $1 \mathrm{~h}$ at room temperature. The membrane was placed in a Vilber Lourmat (Bio-Rad Laboratories, Inc.) for enhanced chemiluminescence development. The density values were determined against the target protein, $\beta$-actin.

Proliferation assay. An MTT assay was performed to determine the rates of proliferation. As the RNAi-YAP-1 transfection group exhibited the most efficient inhibition of YAP-1, cells stably expressing pMagic7.1-Puro/GFP-RNAi-YAP-1 were selected to assess proliferation, and the NC group was used as the control group. Each group was seeded at a low density $\left(2 \times 10^{3}\right.$ cells $\left./ 200 \mu \mathrm{l}\right)$. Blank medium was used to obtain a zero setting. The cells were incubated for 7 days at $37^{\circ} \mathrm{C}$, and the cells were randomly removed each day and the cell numbers were determined using a microplate reader (M450; Bio-Rad Laboratories, Inc.). A proliferation curve was generated, and the doubling time of the cells was calculated using the Patterson formula: $\mathrm{Td}=\mathrm{T} \times \lg 2 / \lg (\mathrm{Nt} / \mathrm{N} 0)$, where $\mathrm{Td}$ indicates the doubling time, $\mathrm{T}$ indicates the number of days, $\mathrm{Nt}$ indicates the number of cells on the final day and NO indicates the number of cells on the first day.

Cell cycle assay. The PC- 3 cells transfected with RNAi-YAP-1, which yielded the highest inhibition ratio, and the NC group were cultured with DMEM/F12 containing $10 \% \mathrm{FBS}$ at $4^{\circ} \mathrm{C}$ for $12 \mathrm{~h}$. The cells were then harvested and fixed with 70\% ethanol overnight at $4^{\circ} \mathrm{C}$. The fixed cells were stained with $50 \mu \mathrm{g} / \mu \mathrm{l}$ propidium iodide (Bioscience, Shanghai, China) and $100 \mu \mathrm{g} / \mu \mathrm{l}$ RNase (Sigma-Aldrich, St. Louis, MO, USA). The cell cycle profiles of the two groups were measured using flow cytometry (FCM; FC 500 Series Flow Cytometry System; Beckman Coulter, Inc., Brea, CA, USA), and the resulting data were analysed.

Apoptosis assay. The cells of the RNAi-YAP-1-transfected group and the NC group were collected and cultured, as above. The cells were harvested and fixed with $70 \%$ ethanol at $4^{\circ} \mathrm{C}$ overnight. The fixed cells were stained with $50 \mu \mathrm{g} / \mu \mathrm{l}$ propidium iodide at $4^{\circ} \mathrm{C}$ for $30 \mathrm{~min}$. The apoptotic profile was determined, 
Table I. Expression of YAP in different tissues.

\begin{tabular}{|c|c|c|c|c|c|c|c|}
\hline \multirow[b]{2}{*}{ Tissue } & \multirow[b]{2}{*}{ Cases (n) } & \multicolumn{4}{|c|}{ Score of YAP intensity } & \multirow[b]{2}{*}{ Positive (\%) } & \multirow[b]{2}{*}{ P-value } \\
\hline & & $0-1$ & 2 & $3-4$ & $5-6$ & & \\
\hline Para-PCa & 15 & 11 & 3 & 1 & 0 & 26.67 & 0.007 \\
\hline $\mathrm{BPH}$ & 15 & 15 & 0 & 0 & 0 & 0 & 0.002 \\
\hline $\mathrm{PCa}$ & 32 & 7 & 2 & 7 & 16 & 78.13 & \\
\hline Total & 62 & 33 & 5 & 8 & 16 & 46.77 & \\
\hline
\end{tabular}

YAP, Yes-associated protein; $\mathrm{PCa}$, prostate cancer; $\mathrm{BPH}$, benign prostatic hyperplasia.

following analysis of the cells by FCM, using a $630 \mathrm{~nm}$ argon ion laser.

Statistical analysis. Statistical analysis was performed using SPSS 18.0 software (SPSS, Inc., Chicago, IL, USA). Data are expressed as the mean \pm standard deviation. A $\chi^{2}$ test was used to compare the prostatic tissues. Student's two-tailed t-test was used to compare between groups. Regression analysis was used to perform correlation analysis. $\mathrm{P}<0.05$ was considered to indicate a statistically significant difference.

\section{Results}

Protein expression of YAP in different prostatic tissues. No positivity for YAP protein (intensity score $\leq 1$ ) were observed in the BPH tissue (0/15; Fig. 1A). In the PCa tissue, $78.13 \%(25 / 32)$ of the samples (intensity score $>5$ ) exhibited positive expression, and the positive areas were primarily located in the cytoplasm and nuclei of the PCa glandular epithelium (Fig. 1B). In the para-PCa tissue, the frequency of samples (intensity score 2-4) was $26.67 \%$ (4/15), and the positive areas were predominantly in the cytoplasm, with a small quantity in the nuclei (Fig. 1C). The percentage of YAP protein expressed in the PCa tissue was significantly higher, compared with the $\mathrm{BPH}$ tissue $(\mathrm{P}=0.002)$ and para-PCa tissue $(\mathrm{P}=0.007$; Table I). Based on the results of the western blot analysis, the expression of YAP was high in the PCa tissue, but low in the BPH tissue and para-PCa tissue (Fig. 1D).

Correlation between the expression of YAP and the clinicopathological grading and staging of $\mathrm{PCa}$. The frequencies of samples with positive expression of YAP among tissues with Gleason scores of $<7,7$, and $\geq 8$ were $50 \%$ (4/8), $72.7 \%(8 / 11)$, and $100 \%(13 / 13)$, respectively, revealing a positive correlation $(\mathrm{P}=0.008$; Fig. 2A). Based on the TNM staging, the samples in which YAP was present exhibited an increasing trend between $\mathrm{T} 1$ and T4. For T1+T2 and T3+T4 stages, the frequencies were $60 \%(9 / 15)$ and $94.1 \%(16 / 17)$, respectively $(\mathrm{P}=0.033$; Fig. 2B). The expression of YAP increased significantly with increasing PSA levels ( $<10 \mathrm{ng} / \mathrm{ml} \mathrm{vs} . \geq 10 \mathrm{ng} / \mathrm{ml}$, $\mathrm{P}=0.0032$; Table II, Fig. 2C).

Plasmid-transfected PC-3 cells. GFP was expressed in the PC-3 cells $48 \mathrm{~h}$ after transfection with the
pMagic7.1-Puro/GFP-RNAi-1, 2, and 3 plasmids, which suggested that each group was transfected successfully (Fig. 3A and B). After 2 weeks of selection with $1 \mathrm{mg} / \mathrm{l}$ puromycin, small clusters of green fluorescent cells were observed (Fig. 3C). Subsequently, three groups of stable YAP interference cell lines, as well as one non-targeted knockdown control cell line, were established from the PC-3 cells (Fig. 3D).

$m R N A$ and protein expression of YAP following transfection. The results of the RT-qPCR analysis demonstrated that the mRNA expression level of YAP in the RNAi transfected cells was markedly, compared with those in the NT and NC groups (Fig. 4A). Decreased protein expression of YAP was also confirmed by western blot analysis (Fig. 4B). The lowest expression level of YAP was observed in the cells transfected with the RNAi-YAP-1 plasmid, which indicated that the RNAi-YAP-1 plasmid had the highest inhibition ratio of the three plasmids and, therefore, the RNAi-YAP-1 plasmid was selected for subsequent investigation.

Interference with RNAi-YAP-1 suppresses PC-3 cell growth. To understand the physiological role of YAP, the expression of endogenous YAP was inhibited in the PC-3 cells. RT-qPCR and western blot analysis revealed that high expression levels of YAP in the PC-3 cells. To determine the effect of the expression of YAP on PC-3 cell growth, proliferation curves of the stable RNAi-YAP-1 interference clones were determined and compared with the corresponding NC (Fig. 5). The two-factor analysis of variance revealed that the difference between the two groups was statistically significant $(\mathrm{P}=0.022)$, based on the optical density value from the third day. The doubling times of the two groups were also significantly different, in which the transfected group exhibited a doubling time of $57.8 \mathrm{~h}$, and the NC group exhibited a doubling time of $23.2 \mathrm{~h}(\mathrm{P}=0.028)$.

Inhibiting YAP dysregulates the cell-cycle of PC-3 cells. To determine whether the knockdown of YAP was sufficient to induce G1/S cell cycle arrest in the PC-3 cells, cell cycle analysis was performed. As shown in Fig. 6, in the transfected group, FCM revealed more cells in the G1 phase (61.4\%) and fewer cells in the S phase (29.4\%), compared with the NC group (G1 phase, 44.8\%; S phase 40.7\%; $\mathrm{P}=0.009$ ). These findings indicated that a dysregulated cell cycle in PC-3 cells 
Table II. Association of YAP with the clinicopathologic features of prostate cancer.

\begin{tabular}{|c|c|c|c|c|c|c|c|}
\hline \multirow[b]{2}{*}{ Feature } & \multirow[b]{2}{*}{ Cases (n) } & \multicolumn{3}{|c|}{ Score of YAP intensity } & \multirow[b]{2}{*}{$5-6$} & \multirow[b]{2}{*}{ Positive (\%) } & \multirow[b]{2}{*}{ P-value } \\
\hline & & $0-1$ & 2 & $3-4$ & & & \\
\hline Gleason grading & & & & & & & 0.008 \\
\hline$<7$ & 8 & 4 & 1 & 3 & 0 & 50 & \\
\hline 7 & 11 & 3 & 1 & 2 & 5 & 72.7 & \\
\hline $8-10$ & 13 & 0 & 0 & 2 & 11 & 100 & \\
\hline TNM stage & & & & & & & 0.033 \\
\hline $\mathrm{T} 1+\mathrm{T} 2$ & 15 & 6 & 1 & 3 & 5 & 60 & \\
\hline $\mathrm{T} 3+\mathrm{T} 4$ & 17 & 1 & 1 & 4 & 11 & 94.1 & \\
\hline PSA level (ng/ml) & & & & & & & 0.032 \\
\hline$<10$ & 11 & 5 & 1 & 3 & 2 & 54.5 & \\
\hline$\geq 10$ & 21 & 2 & 1 & 4 & 14 & 90.5 & \\
\hline
\end{tabular}

YAP, Yes-associated protein; TNM, tumour-node-metastasis; PSA, prostate specific antigen.

A

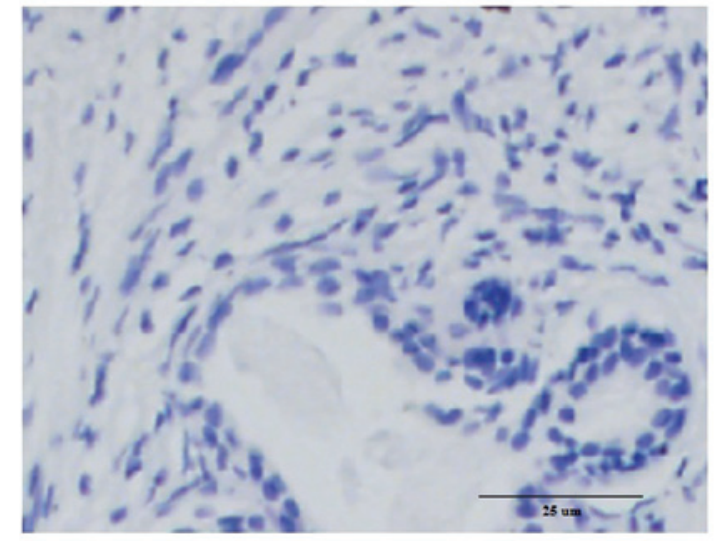

C

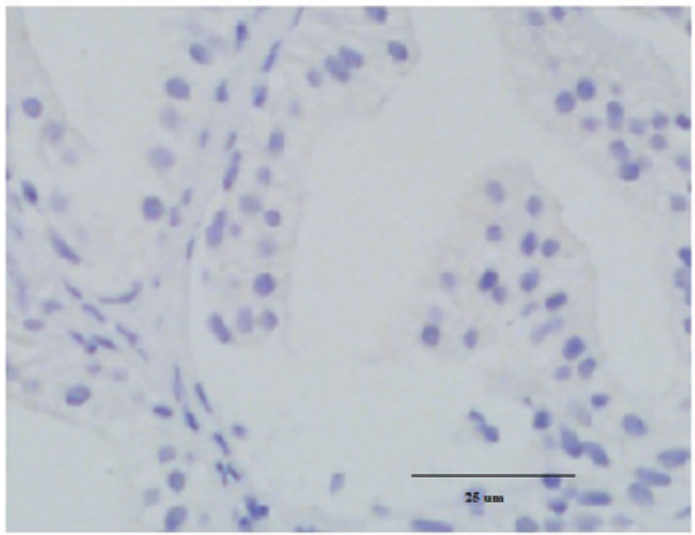

B

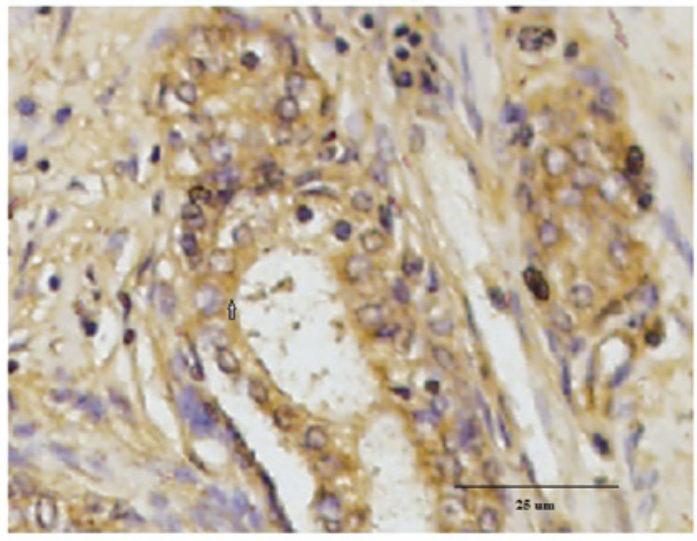

D
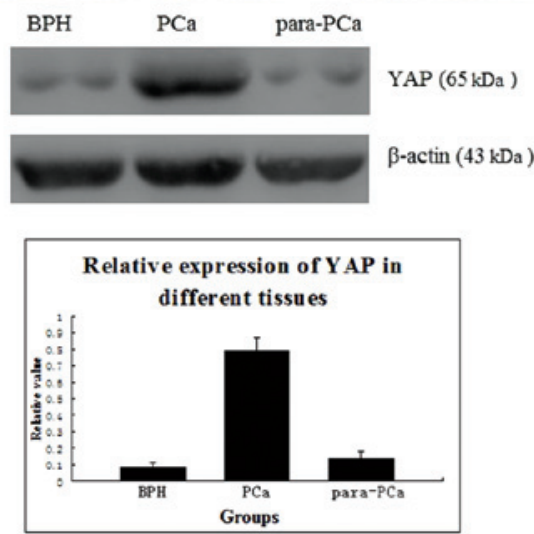

Figure 1. Immunohistochemical analysis of protein expression in 32 samples of PCa tissue, 15 samples of BPH tissue and 15 samples of para-PCa tissue. (A) Protein expression of YAP in BPH (magnification, x400); (B) Overexpression of YAP protein in PCa (magnification, x400); (C) Protein expression of YAP in para-PCa tissue (magnification, $\mathrm{x} 400$ ); The difference in the expression of YAP was significant between $\mathrm{PCa}$ and $\mathrm{BPH}(\mathrm{P}=0.007)$, and between $\mathrm{PCa}$ and para-PCa ( $\mathrm{P}=0.002)$; (D) Expression of YAP in different prostatic cells, analysed using western blotting, in all tissues. Data are expressed as the mean \pm standard deviation. PCa, prostate cancer; YAP, Yes-associated protein; BPH, benign prostatic hyperplasia.

subjected to YAP-knockdown, led to arrest of cells in the G1 phase.

Knockdown of YAP induces apoptosis of PC-3 cells. In order to understand the reason for the growth suppression observed in the transfected PC-3 cells, an apoptosis assay was performed. Apoptosis of the cells in the NC group and transfected group was detected using FCM, which revealed that the apoptosis of PC-3 cells was induced by YAP knockdown. The difference in the apoptotic rate between 
A

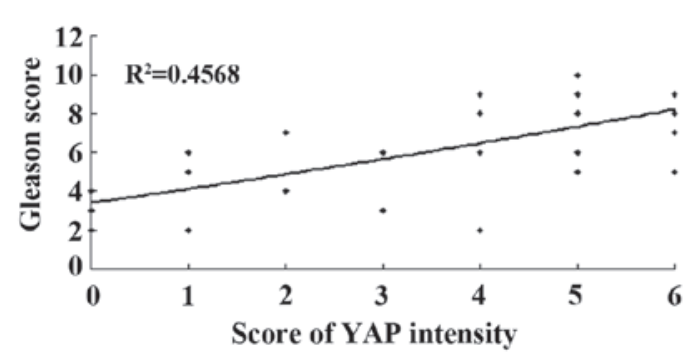

C

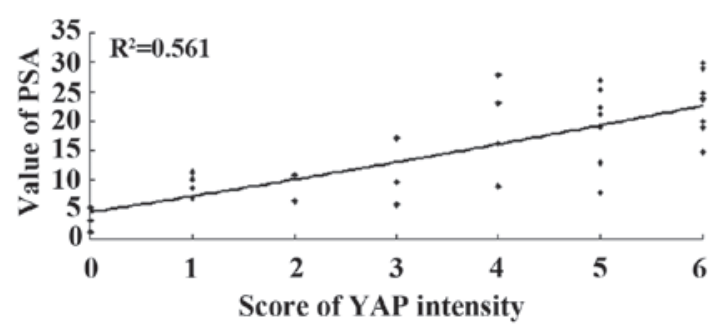

B

\section{Correlation between YAP and TNM stage}

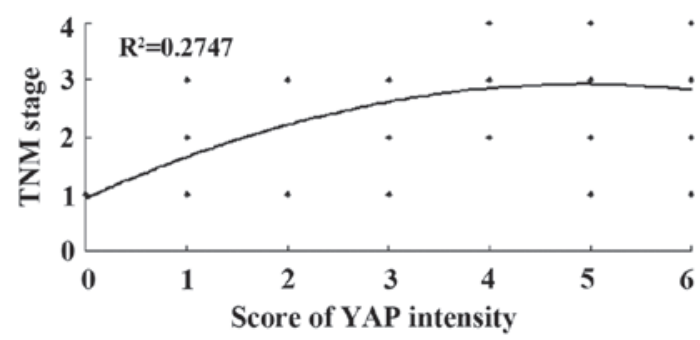

Figure 2. Correlation between YAP and Gleason score, TNM stage and PSA level using curve estimation regression analysis. (A) Correlation between YAP and Gleason score, in which a positive trend was observed between them. (B) Correlation between YAP and TNM stage. The integrating degree was not perfect between the linear and quadratic or cubic curve. (C) A positive correlation was also observed between YAP and PSA levels. PSA, prostate specific antigen; YAP, Yes-associated protein; TNM, tumour-node-metastasis.

A

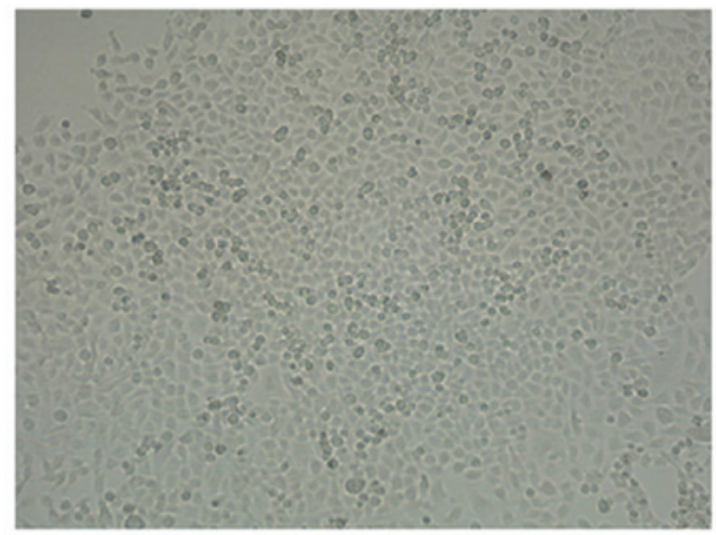

C

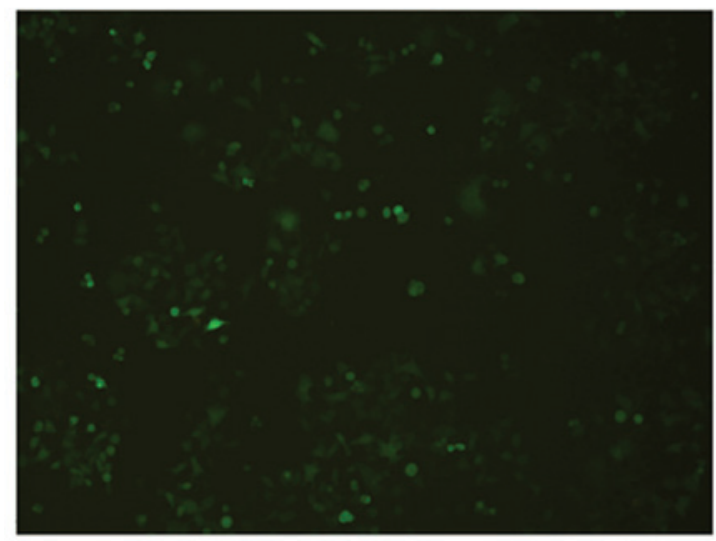

B

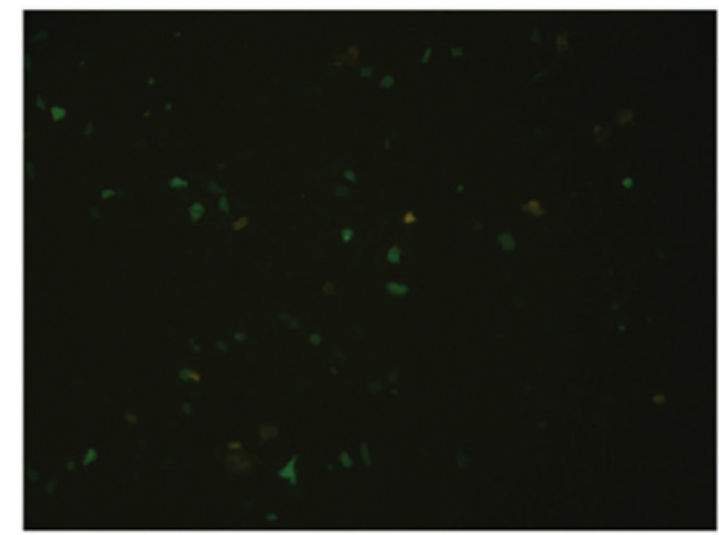

D

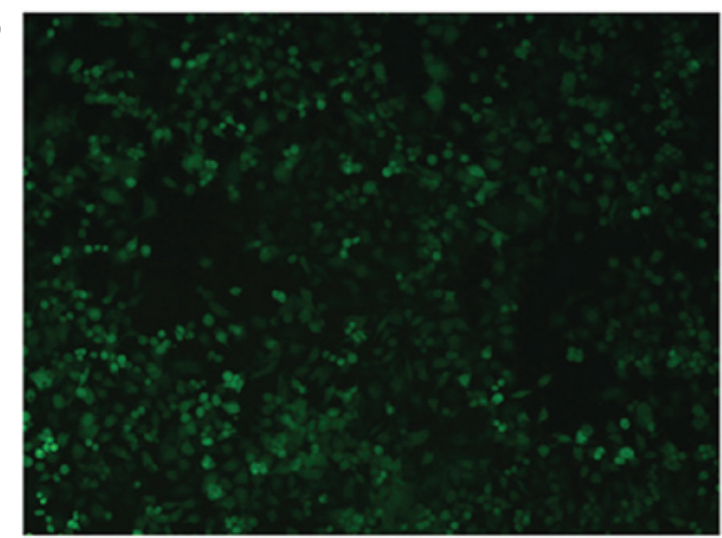

Figure 3. Transfection assay using a fluorescent plasmid. The expression of green fluorescent protein with interference plasmid transfected PC-3 cells was assessed. (A) Imaging under an inverted microscope (magnification, x200) following transfection for $48 \mathrm{~h}$; (B) Cells following transfection for $48 \mathrm{~h}$ under an inverted fluorescence microscope (magnification, x200); (C) Fluorescent cell screening 2 weeks following treatment with puromycin (magnification, x200); (D) Fluorescent cell screening after 4 weeks (magnification, $x 200$ ), which were considered to be stably expressing green fluorescence. 
M NT NC Ri-1 Ri-2 Ri-3
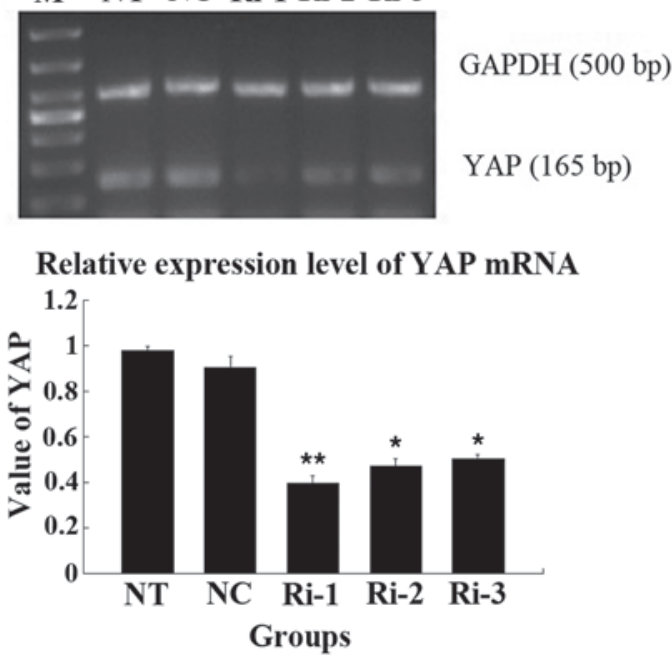

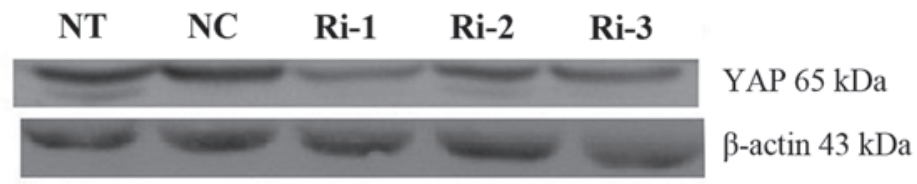

Relative expression of YAP protein

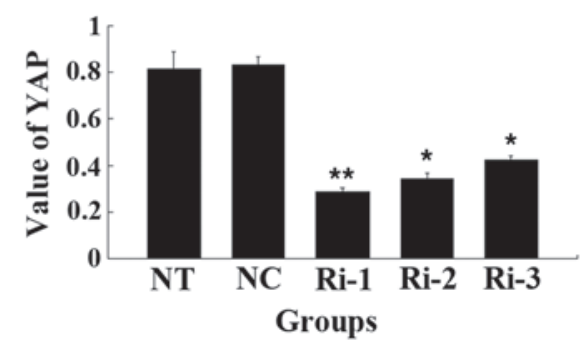

Figure 4. Different expression levels of the YAP in the stable cells following RNA-YAP interference-1, 2, 3 plasmid transfection. (A) mRNA expression of YAP in the cells of different groups following transfection with RNAi-YAP-1, 2 and 3; (B) Protein expression of YAP in the cells of different groups following transfection with RNAi-YAP-1, 2 and 3. RNAi-YAP-1 had the most marked effect on YAP inhibition, and RNAi-YAP-2 and RNAi-YAP-3 exhibited weaker effects. Therefore, the RNAi-YAP-1 plasmid was used for the subsequent investigations. Data are expressed as the mean \pm standard deviation; ${ }^{*} \mathrm{P}<0.05,{ }^{* *} \mathrm{P}<0.01$ vs. control. YAP, yes-associated protein; NC, negative control; NT, non-transfected; Ri, RNA-YAP.

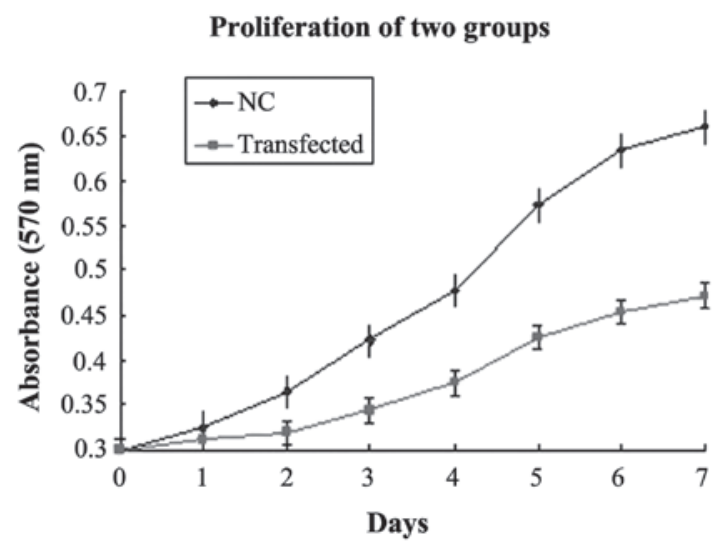

Figure 5. Analysis of proliferation of cells in different groups using an MTT assay. Absorbance in yes-associated protein interference stable clones and $\mathrm{NC}$ group cells were counted for 7 days. Growth curves were plotted to assess the growth pattern of each cell line Data are expressed as the mean \pm standard deviation. ( $\mathrm{P}=0.022)$. NC, negative control.

the $\mathrm{NC}$ and transfected cells was statistically significant ( $\mathrm{P}=0.002$; Fig. 7).

Knockdown of YAP inhibits the $M R N A$ and protein expression of TEAD1. TEAD1 is a downstream gene of YAP in the Hippo signalling pathway $(5,11)$. It has been hypothesized that the activity of the TEAD1 gene is regulated by the YAP protein, and binds with the TEAD1 combining domain to form a compound, which generates TEAD1 protein (12). In the present study, the PC-3 cells transfected with RNAi-YAP-1, the mRNA expression was significantly lower, compared with those in the NT and NC groups $(\mathrm{P}=0.001$; Fig. 8A). A western blot assay was also performed, which demonstrated that the protein expression of TEAD1 was also lower in the cells transfected with RNAi-YAP-1 (P=0.005; Fig. 8B). By contrast, YAP was inhibited successfully following TEAD1 inhibition.

\section{Discussion}

Carcinoma results from the loss of normal cell communication, which causes unregulated cell proliferation, migration and the inhibition of apoptosis (31). The Hippo pathway is important in the regulation of tumour cells and tissue growth (32). The overexpression of YAP, which is the core component in the Hippo-YAP pathway, increased organ size and caused cancer in transgenic mice (33). The expression of YAP has been significantly associated with tumour metastasis, grade and stage $(34,35)$. YAP depletion suppresses the expression of cell cycle-promoting genes in tumour cells and regulates tumour growth and metastasis $(32,36)$. There is also evidence that YAP is a valid molecular target (37). In the present study, it was demonstrated that YAP closely correlates with PCa, and that the knockdown of YAP is essential in growth suppression and cell cycle dysregulation in CRPC cells.

Initially, the present study demonstrated that the protein level of YAP protein was correlated with YAP biological activities, with certain traits that have been associated with PCa staging and grading. Para-PCa and BPH tissues had low frequencies of YAP-positive cells, whereas PCa tissue had high frequencies $(\mathrm{P}=0.008)$. In addition, the frequency of YAP-positive cells increased significantly between low-grade $\mathrm{PCa}$ and high-grade $\mathrm{PCa}(\mathrm{P}=0.033)$. It was suggested that the YAP activity was closely-associated with progression between well-differentiated and high-grade tumours. YAP activity is a clinically relevant tool to predict an increasing proclivity to develop metastases and higher PSA levels $(\mathrm{P}=0.0032)$. Correlation analysis demonstrated that the protein expression of YAP was significantly correlated with the mRNA expression, indicating that the YAP 
A

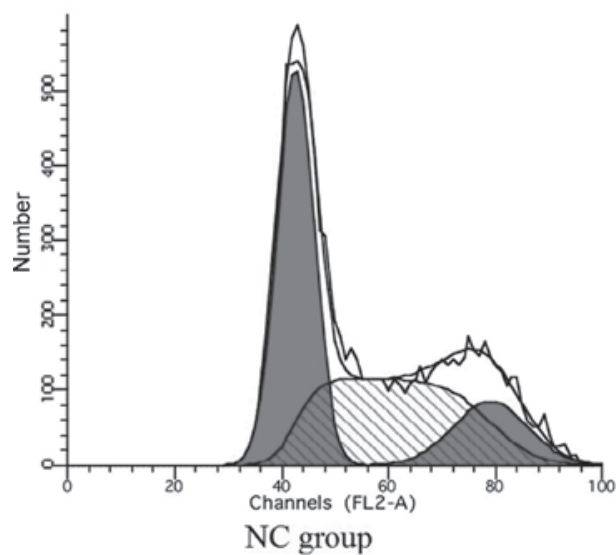

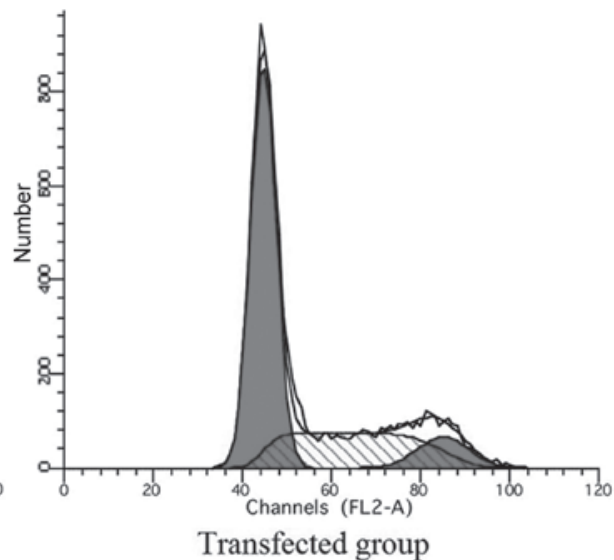

C Phases of cell-cycle

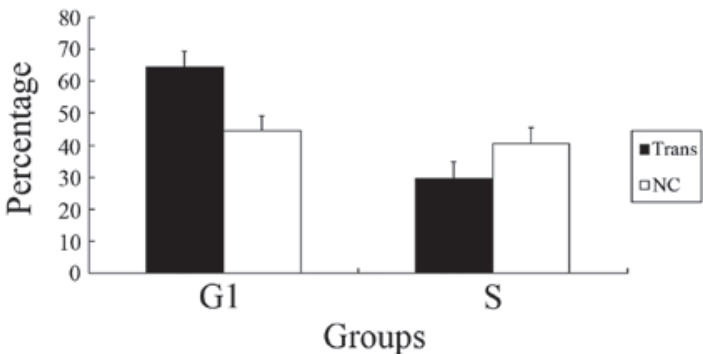

Figure 6. (A) Following treatment with RNaseA, the cells were stained with propidium iodide and flow cytometry was performed to analyse the cell-cycle of the NC group. (B) Following Yap knockdown, the cell-cycle of the PC-3 cells transfected with the RNAi-YAP-1 plasmid was arrested at the G1 stage (C) Bar chart of the percentages of cells in the G1 and S stages in the transfected group and NC group. A higher percentage of cells in the transfected group were arrested at the G1 stage, compared with the NC group. Data are expressed as the mean \pm standard deviation. NC, negative control; YAP, yes-associated protein; Trans, transfected.

A

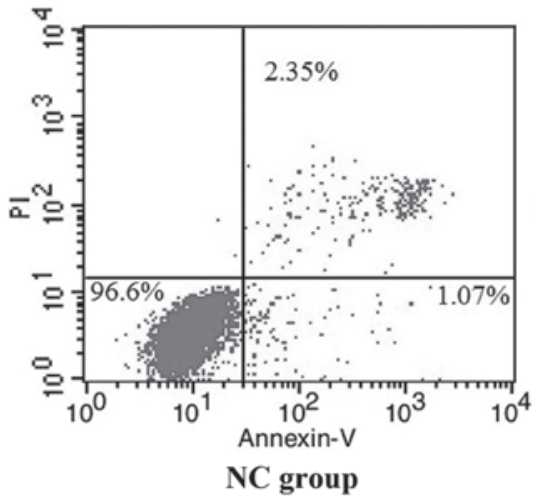

B

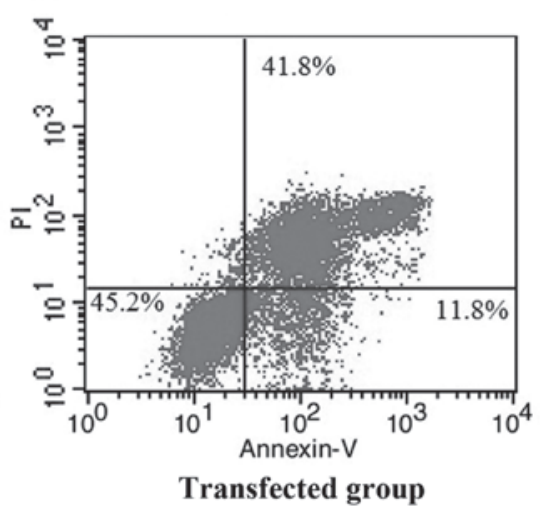

C

Apoptosis of cells

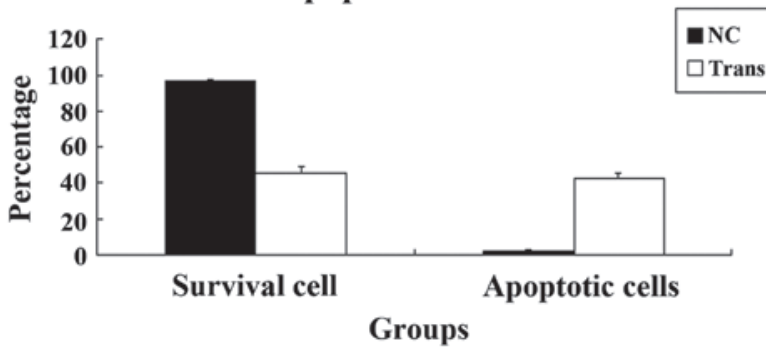

Figure 7. (A) Apoptosis of the NC group, detected using flow cytometry; (B) Apotosis of cells transfected with the RNAi-YAP-1 plasmid. The difference in apoptosis between the $\mathrm{NC}$ group and transfected group was significant $(\mathrm{P}=0.002)$. (C) Bar chart demonstrating the results of the apoptosis assay in the $\mathrm{NC}$ group and transfected group. Data are expressed as the mean \pm standard deviation. NC, negative control; YAP, yes-associated protein; PI, propidium iodide; Trans, transfected. 


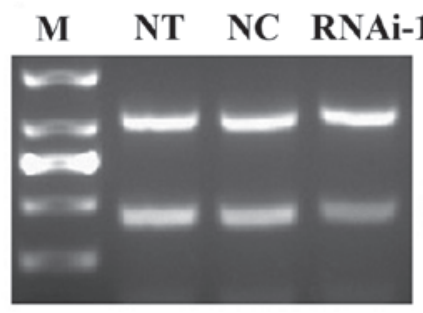

Relative expression of TEAD1 mRNA

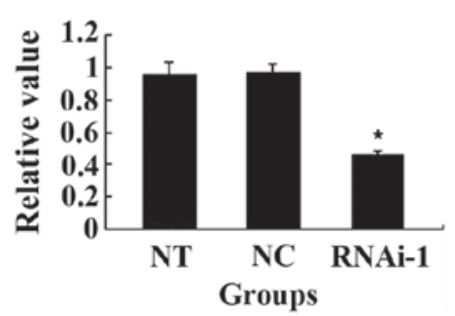

B

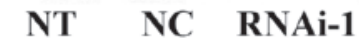

GAPDH (500 bp)

TEADl (280 bp)

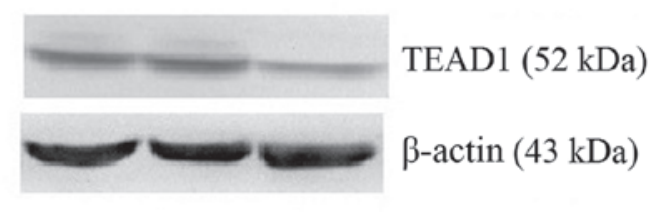

Relative expression of

TEAD1 protein

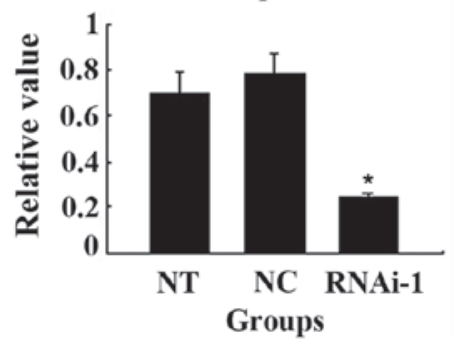

Figure 8. mRNA and protein expression of TEAD1 in the different groups of cells. (A) mRNA expression of TEAD1 was lower in the RNAi-YAP-1 group, determined using reverse transcription-quantitative polymerase chain reaction. (B) Protein expression of TEAD1 was lower in the RNAi-YAP-1 group, determined using western blotting; The mRNA and protein expression levels of TEAD1 were inhibited following YAP knockdown. Data are expressed as the mean \pm standard deviation; ${ }^{*} \mathrm{P}<0.05,{ }^{* *} \mathrm{P}<0.001$ vs. control. RNAi, RNA interference; YAP, yes-associated protein; NT, non-transfected; NC, negative control; TEAD1, TEA domain family member $1 ; \mathrm{M}$, marker.

gene transcription and protein expression were coordinated. In the earlier stages of $\mathrm{PCa}$, high levels of expression of YAP were observed, and increased expression of YAP was accompanied by increased stages of malignancy. The YAP gene is a tumour-specific gene, which may be involved in the occurrence and development of PCa.

It is known that current treatments, including docetaxel only provide a modest increase in survival rates of patients with CRPC, and the majority of patients eventually progress due to drug resistance (38). Therefore, the present study investigated PC-3 cells to determine whether there an association exists between YAP and CRPC, and the results demonstrated that YAP was expressed in PC-3 cells enabling further investigations.

The potency of the Hippo pathway in driving tissue growth appears to reside in its ability to coordinately stimulate cell proliferation and suppress apoptosis (39). A key goal is to understand how this coordinated control is achieved. The results of the present study demonstrated that pMagic7.1-Puro/GFP-RNAi can target YAP in PC-3 cells. Specific RNAi vectors targeting human YAP were transfected into PC-3 cells with liposomes, and cell lines that stably expressrf pMagic7.1-Puro/GFP-RNAi-YAP were obtained, as indicated by the expression of GFP. Using RT-qPCR and western blotting, the present study demonstrated that the mRNA and protein expression of YAP were inhibited effectively in these cells. Using FCM and an MTT colorimetric assay, it was observed that the proliferation of the PC-3 cells decreased significantly, and cell-cycle was arrested at the G1 stage in vitro when the YAP gene was suppressed. This result was consistent with previous findings associated with YAP in the majority of other tumour types (40). The mRNA and protein expression of TEAD1 was also inhibited following the inhibited YAP.
In conclusion, the present study demonstrated the efficacy of potent YAP knockdown in inhibiting the growth and inducing apoptosis of CRPC cells in vitro. The inhibitory effect induced by the deactivation of YAP may be explained, in part, by inactivation of the Hippo-YAP pathway in the CRPC cells. An important direction for future investigations is to elucidate how YAP is involved in the development and progression of CRPC and how it regulates the proliferation and cell-cycle of CRPC cells. This may be facilitated by investigating the mechanism underlying the Hippo-YAP pathway in CRPC. The results obtained in the present study demonstrate the importance of YAP activity in CRPC cell biology and indicate that further analysis of the molecular basis for its tumour suppressive role is warranted. Understanding these mechanisms may contribute to the development of preventive and therapeutic strategies for the treatment of CRPC.

\section{Acknowledgements}

The present study was supported by the Natural Science Foundation of China (grant no. 30972999). The contents of the present study are solely the responsibility of the authors and do not necessarily represent the official views of the Natural Science Foundation of China.

\section{References}

1. Conlon I and Raff M: Size control in animal development. Cell 96: 235-244, 1999.

2. Wu S, Huang J, Dong J and Pan D: Hippo encodes a Ste-20 family protein kinase that restricts cell proliferation and promotes apoptosis in conjunction with Salvador and warts. Cell 114: 445-456, 2003

3. Zhao B, Wei X, Li W, Udan RS, Yang Q, Kim J, Xie J, Ikenoue T, Yu J, Li L, et al: Inactivation of YAP oncoprotein by the Hippo pathway is involved in cell contact inhibition and tissue growth control. Genes Dev 21: 2747-2761, 2007. 
4. Chen L, Chan SW, Zhang X, Walsh M, Lim CJ, Hong W and Song H: Structural basis of YAP recognition by TEAD4 in the hippo pathway. Genes Dev 24: 290-300, 2010.

5. Huang J, Wu S, Barrera J, Matthews K and Pan D: The Hippo signaling pathway coordinately regulates cell proliferation and apoptosis by inactivating yorkie, the Drosophila Homolog of YAP. Cell 122: 421-434, 2005.

6. Nolo R, Morrison CM, Tao C, Zhang X and Halder G: The bantam microRNA is a target of the hippo tumor-suppressor pathway. Curr Biol 16: 1895-1904, 2006.

7. Thompson BJ and Cohen SM: The Hippo pathway regulates the bantam microRNA to control cell proliferation and apoptosis in Drosophila. Cell 126: 767-774, 2006.

8. Bertini E, Oka T, Sudol M, Strano S and Blandino G: YAP: At the crossroad between transformation and tumor suppression. Cell Cycle 8: 49-57, 2009.

9. Overholtzer M, Zhang J, Smolen GA, Muir B, Li W, Sgroi DC, Deng CX, Brugge JS and Haber DA: Transforming properties of YAP, a candidate oncogene on the chromosome 11q22 amplicon. Proc Natl Acad Sci USA 103: 12405-12410, 2006.

10. Zender L, Spector MS, Xue W, Flemming P, Cordon-Cardo C, Silke J,Fan ST, Luk JM, Wigler M, Hannon GJ, et al: Identification and validation of oncogenes in liver cancer using an integrative oncogenomic approach. Cell 125: 1253-1267, 2006.

11. Sawada A, Kiyonari H, Ukita K, Nishioka N, Imuta Y and Sasaki H: Redundant roles of Tead1 and Tead2 in notochord development and the regulation of cell proliferation and survival. Mol Cell Biol 28: 3177-3189, 2008

12. Zhang L, Ren F, Zhang Q, Chen Y, Wang B and Jiang J: The TEAD/TEF family of transcription factor Scalloped mediates hippo signaling in organ size control. Dev Cell 14: 377-387, 2008

13. Ota $M$ and Sasaki H: Mammalian Tead proteins regulate cell proliferation and contact inhibition as transcriptional mediators of hippo signaling. Development 135: 4059-4069, 2008.

14. Zhao B, Ye X, Yu J, Li L, Li W, Li S, Yu J, Lin JD, Wang CY, Chinnaiyan AM, et al: TEAD mediates YAP-dependent gene induction and growth control. Genes Dev 22: 1962-1971, 2008.

15. Dupont S, Morsut L, Aragona M,Enzo E, Giulitti S, Cordenonsi M, Zanconato F, Le Digabel J, Forcato M, Bicciato S, et al: Role of YAP TAZ in mechanotransduction. Nature 474: 179-183, 2011.

16. Chan SW, Lim CJ, Loo LS, Chong YF, Huang C and Hong W: TEADs mediate nuclear retention of TAZ to promote oncogenic transformation. J Bio Chem 284: 14347-14358, 2009.

17. Jemal A, Bray F, Center MM, Ferlay J, Ward E and Forman D Global Cancer Statistics. Ca Cancer J Clin 61: 69-90, 2011.

18. Katoh M: Function and cancer genomics of FAT family genes (review). Int J Oncol 41: 1913-1918, 2012.

19. Lu L, Li Y, Kim SM, Bossuyt W, Liu P, Qiu Q, Wang Y, Halder G Finegold MJ, Lee JS, et al: Hippo signaling is a potent in vivo growth and tumor suppressor pathway in the mammalian liver. Proc Natl Acad Sci USA 107: 1437-1442, 2010.

20. Zhou Z, Zhu JS, Xu ZP and Zhang Q: Lentiviral vector-mediated siRNA knockdown of the YAP gene inhibits growth and induces aoptosis in the SGC7901 gastric cancer cell line. Mol Med Rep 4: 1075-1082, 2011

21. Li W, Wang L, Katoh H, Liu R, Zheng P and Liu Y: Identification of a tumor suppressor relay between the FOXP3 and the Hippo pathways in breast and prostate cancers. Cancer Res 71 2162-2171, 2011.

22. Zagurovskaya M, Shareef MM, Das A, Reeves A, Gupta S, Sudol M, Bedford MT, Prichard J, Mohiuddin M and Ahmed MM: EGR-1 forms a complex with YAP-1 and up regulates Bax expression in irradiated prostate carcinoma cells. Oncogene 28 : 1121-1131, 2009.

23. Tapon N, Harvey KF, Bell DW, Wahrer DC, Schiripo TA, Haber D and Hariharan IK: Salvador promotes both cell cycle exit and apoptosis in Drosophila and is mutated in human cancer cell lines. Cell 110: 467-478, 2002.
24. Nishioka N, Inoue K, Adachi K, Kiyonari H, Ota M, Ralston A, Yabuta N, Hirahara S, Stephenson RO, Ogonuki N, et al: The Hippo signaling pathway components Lats and Yap pattern Tead4 activity to distinguish mouse trophectoderm from inner cell mass. Dev Cell 16: 398-410, 2009.

25. Berney DM: Low Gleason score prostatic adenocarcinomas are no longer viable entities. Histopathology 50: 683-690, 2007.

26. Na Y, Ye Z and Sun G: Diagnosis and Treatment Guideline of Chinese Urology Diseases. People's Medical Publishing House, Beijing, p52-53, 2011 (In Chinese).

27. Hudson TS, Perkins SN, Hursting SD, Young HA, Kim YS, Wang TC and Wang TT: Inhibition of androgen-responsive LNCaP prostate cancer cell tumor xenograft growth by dietary phenethyl isothiocyanate correlates with decreased angiogenesis and inhibition of cell attachment. Int J Oncol 40: 1113-1121, 2012.

28. Sheng X, Li Z, Wang DL, Li WB, Luo Z, Chen KH, Cao JJ, Yu C and Liu WJ: Isolation and enrichment of PC-3 prostate cancer stem-like cells using MACS and serum-free medium. Oncol Lett 5: 787-792, 2013.

29. Wang DL, Lan JH, Chen L, Huang B, Li Z, Zhao XM, Ma Q, Sheng X, Li WB and Tang WX: Integrin-linked kinase functions as a tumor promoter in bladder transitional cell carcinoma. Asian Pac J Cancer Prev 13: 2799-2806, 2012.

30. Okamoto Y, Ohkubo T, Ikebe T and Yamazaki J: Blockade of TRPM8 activity reduces the invasion potential of oral squamous carcinoma cell lines. Int J Oncol 40: 1431-1440, 2012.

31. De Ganck A, De Corte V,BruyneelE, Bracke M, Vandekerckhove J and Gettemans J: Down-regulation of myopodin expression reduces invasion and motility of PC-3 prostate cancer cells. Int J Oncol 34: 1403-1409, 2009.

32. Lamar JM, Stern P, Liu H, Schindler JW, Jiang ZG and Hynes RO: The Hippo pathway target, YAP, promotes metastasis through its TEAD-interaction domain. Proc Natl Acad Sci USA 109: E2441-E2450, 2012.

33. Dong J, Feldmann G, Huang J, Wu S, Zhang N, Comerford SA, Gayyed MF, Anders RA, Maitra A and Pan D: Elucidation of a universal size-control mechanism in Drosophila and mammals. Cell 130: 1120-1133, 2007.

34. Zhao B, Li L, Wang L, Wang CY, Yu J and Guan KL: Cell detachment activates the Hippo pathway via cytoskeleton reorganization to induce anoikis. Genes Dev 26: 54-68, 2012.

35. Yamazaki H, Nishiyama K, Tanaka E, Maeda O, Meguro N, Kinouchi T, Usami M, Kakimoto K, Ono Y and Nishimura T: Reduction of irradiation volume and toxicities with 3-D radiotherapy planning over conventional radiotherapy for prostate cancer treated with long-term hormonal therapy. Anticancer Res 28: 3913-3920, 2008

36. Mizuno T, Murakami H, Fujii M, Ishiguro F, Tanaka I, Kondo Y, Akatsuka S, Toyokuni $\mathrm{S}$, Yokoi $\mathrm{K}$, Osada $\mathrm{H}$, et al: YAP induces malignant mesothelioma cell proliferation by up regulating transcription of cell cycle-promoting genes. Oncogene 31: 5117-5122, 2012.

37. Cordenonsi M, Zanconato F, Azzolin L, Forcato M, Rosato A, Frasson C, Inui M, Montagner M, Parenti AR, Poletti A, et al: The Hippo transducer TAZ confers cancer stem cell-related traits on breast cancer cells. Cell 147: 759-772, 2011.

38. Rick FG, Schally AV, Szalontay L, Block NL, Szepeshazi K, Nadji M, Zarandi M, Hohla F, Buchholz S and Seitz S: Antagonists of growth hormone-releasing hormone inhibit growth of androgen-independent prostate cancer through inactivation of ERK and Akt kinases. Proc Natl Acad Sci USA 109: 1655-1660, 2012

39. Zhang X, Grusche FA and Harvey KF: Control of tissue growth and cell transformation by the Salvador/Warts/Hippo pathway. PLoS One 7: e31994, 2012.

40. Xu MZ, Yao TJ, Lee NP, Ng IO, Chan YT, Zender L, Lowe SW, Poon RT and Luk JM: Yes-associated protein is an independent prognostic marker in hepatocellular carcinoma. Cancer 115: 4576-4585, 2009 